\title{
A Comparison of Haemodynamic Effects and Safety between Domestic Levosimendan versus Dobutamine for Hospitalized Patients with Acute Decompensated Heart Failure
}

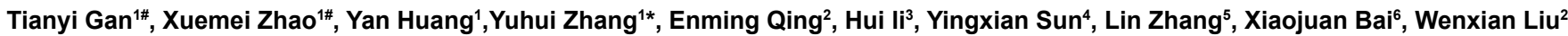
Yinong Jiang ${ }^{7}$, Peng Qu ${ }^{8}$, Bingqi Wei ${ }^{1}$, Qiong Zhou ${ }^{1}$, Shiming Ji ${ }^{1}$ and Jian Zhang ${ }^{1}$

${ }^{1}$ State Key Laboratory of Cardiovascular Disease, Heart Failure Center Fuwai Hospital, National Center for Cardiovascular Diseases, Chinese Academy of Medical Sciences and Peking Union Medical College, Beijing, China

${ }^{2}$ Department of Cardiology, Beijing Anzhen Hospital, Capital Medical University, Beijing, China

${ }^{3}$ Daqing General Hospital Group Oilfield General Hospital, Daqing, China

${ }^{4}$ Department of Cardiology, Shengjing Hospital of China Medical University, Shenyang, China

${ }^{5}$ Department of Cardiology, Beijing Chaoyang Hospital, Capital Medical University, Beijing, China

${ }^{6}$ Department of Cardiology, The First Hospital of China Medical University, Shenyang, China

${ }^{7}$ Department of Cardiology, First Affiliated Hospital of Dalian Medical University, Dalian, China

${ }^{8}$ Department of Cardiology, Second Affiliated Hospital of Dalian Medical University, Dalian, China

\#Tianyi Gan and Xuemei Zhao are co-first authors.

\begin{abstract}
Aims: This study was a phase II trial of efficacy and safety of intravenous treatment with domestic levosimendan versus dobutamine in hospitalized patients with acute decompensated heart failure (ADHF).

Methods and results: This was a multicentre, positive-controlled, prospective randomized open-label blinded study. A total of 228 ADHF patients from 8 medical centres were received $24 \mathrm{~h}$ intravenous domestic levosimendan $(n=114)$ or dobutamine $(n=114)$ therapy. SWAN-GANZ catheter was applied for patients with Pulmonary Capillary Wedge Pressure (PCWP) $\geq 15 \mathrm{mmHg}$ and Cardiac Index $(\mathrm{Cl}) \leq 2.5 \mathrm{~L} / \mathrm{min} / \mathrm{m} 2(\mathrm{n}=39$ each). Compared with baseline level, left ventricular ejection fraction (LVEF) increased at $24 \mathrm{~h}$ in both groups $(31.56 \%$ versus $28.44 \%, P<0.01)$. The change rate of LVEF at $24 \mathrm{~h}$ was similar between two groups $(10.9 \%$ versus $12.7 \%, \mathrm{P}>0.05)$. The change rate of PCWP at $24 \mathrm{~h}$ was remarkably greater in levosimendan group than in dobutamine group $(-38.7 \%$ versus $-23.9 \%$, $\mathrm{P}<0.05)$. The change rate of NT-proBNP level at 3 days was also more remarkable in levosimendan group than in dubotamine group $(-22.4 \%$ versus $-8.6 \%, \mathrm{P}<0.01)$. The incidences of adverse reactions and events were similar between two groups.
\end{abstract}

Conclusion: In patients with ADHF, domestic levosimendan improved haemodynamic performance and NT-proBNP effectively than dobutamine. LVEF improvement was similar between domestic levosimendan and dobutamine. Tolerability and safety were similar between domestic levosimendan and dobutamine.

Keywords: Levosimendan, Dobutamine; Acute decompensated heart failure; Haemodynamic effect

Abbreviations: ADHF: Acute Decompensated Heart Failure; NYHA: New York Heart Association; CI: Cardiac Index; PCWP: Pulmonary Capillary Wedge Pressure; SBP: Systolic Blood Pressure; DLEs: Dose-Limiting Events; BNP: B-type natriuretic peptide; CO: Cardiac Output; PAWP: Pulmonary Average Mean Pressure; SV: Stoke Volume; SVR: Systemic Vascular Resistance; LVESD: Left Ventricular End-Systolic Diameter; LVEDD: Left Ventricular End-Diastolic Diameter; cAMP: Cyclic Adenosine Monophosphate

\section{Introduction}

Levosimendan is a new inotropic agent and calcium sensitizer which has been found to increase myocardial contractility via a sensitization of cardiac troponin C to calcium, to produce vasodilatation and cardioprotection by opening scarolemmal and mitochondrial ATP-sensitive potassium channels in vascular smooth muscle cells respectively and to inhibit phosphodiesterase type III [1]. Now, levosimendan has been approved to treat Acute Decompensated Heart Failure (ADHF) in guidelines and widely used in clinical practice for the treatment of heart failure in different settings since year 2000. This is a phase II trial of efficacy and safety of intravenous treatment with domestic levosimendan produced by Chengdu Shengnuo Biopharm
CO. Ltd. versusdobutamine in acute decompensated HF patients.

\section{Methods}

\section{Study population}

228 patients between 18-75 years old with ADHF of ischemic or nonischemic origin, in New York Heart Association (NYHA) class III-IV, with left ventricular ejection fraction $(\mathrm{LVEF}) \leq 40 \%$, were enrolled in 8 medical centers in China including Fuwai Hospital. This study complied with the Declaration of Helsinki, the research protocol had approved by locally appointed ethics committee and that informed consents had

*Corresponding author: Yuhui Zhang, State Key Laboratory of Cardiovascular Disease, Heart Failure Center Fuwai Hospital, National Center for Cardiovascular Diseases, Chinese Academy of Medical Sciences and Peking Union Medica College, Beijing, China, Tel: +86-15901314243; Fax: +86-10-88396180; E-mail: yuhuizhangjoy@163.com

Received April 25, 2016; Accepted June 18, 2016; Published June 23, 2016

Citation: Gan T, Zhao X, Huang Y, Zhang Y, Qing E, et al. (2016) A Comparison of Haemodynamic Effects and Safety between Domestic Levosimendan versus Dobutamine for Hospitalized Patients with Acute Decompensated Heart Failure. Cardiovasc Pharm Open Access 5: 186. doi:10.4172/2329-6607.1000186

Copyright: (C) 2016 Gan T, et al. This is an open-access article distributed under the terms of the Creative Commons Attribution License, which permits unrestricted use, distribution, and reproduction in any medium, provided the original author and source are credited. 
Citation: Gan T, Zhao X, Huang Y, Zhang Y, Qing E, et al. (2016) A Comparison of Haemodynamic Effects and Safety between Domestic Levosimendan versus Dobutamine for Hospitalized Patients with Acute Decompensated Heart Failure. Cardiovasc Pharm Open Access 5 : 186. doi:10.4172/2329-6607.1000186

Page 2 of 5

been obtained from the subjects (or their guardians) from April 2007 to June 2009. All patients were randomized into levosimendan group (114 patients) and dobutamine group (114 patients), among them 78 patients were tested by Swan-Ganz catheterizationwith a Cardiac Index (CI) $\leq 2.5 \mathrm{~L} / \mathrm{min} / \mathrm{m}$ and pulmonary capillary wedge pressure (PCWP) $\geq 15 \mathrm{mmHg}$ and 39 patients with Swan-Ganz catheterization test in each group.

The main exclusion criteria were systolic blood pressure (SBP) $>180 \mathrm{mmHg}$ or $<90 \mathrm{mmHg}$, malignant arrhythmia, valvular heart disease, hypertrophic and restrictive cardiomyopathy, acute coronary syndrome during 1 week before baseline, severe liver or renal dysfunction, severe pulmonary disease and participating in other clinical trials during 3 months before baseline. During the trial, 3 patients $(1.3 \%)$ were eliminated, 1 patient $(0.4 \%)$ in levosimendan group and 2 patients $(0.8 \%)$ in dobutamine group. 12 patients $(5.3 \%)$ were omitted, 5 patients (2.2\%) in levosimendan group and 7 patients (3.1\%) in dobutamine group. The main causes were patients lost to follow or cannot meet the inclusion criteria.

\section{Study design}

All patients received optimized conventional treatment for HF. Levosimendan group: Levosimendan (Chengdu Shengnuo Biopharm CO. Ltd. $12.5 \mathrm{mg} / 5 \mathrm{~mL}$. Lot number: 061201) was firstly administered as an initial loading dose of $12 \mu \mathrm{g} / \mathrm{kg}$ delivered over $10 \mathrm{~min}$ and then followed by a continuous intravenous infusion of $0.1 \mu \mathrm{g} / \mathrm{kg} / \mathrm{min}$ for 2 hours. The levosimendan infusion rate was increased to $0.2 \mu \mathrm{g} / \mathrm{kg} /$ min for further 22 hours if no Dose-Limiting Events (DLEs) occurred. Dobutamine group: Dobutamine (Shanghai No.1 Biochemical \& Pharmaceutical Co. Ltd. $20 \mathrm{mg} / 2 \mathrm{~mL}$, Lot number: 070402) was administrated as a continuous infusion without a loading dose, beginning at a rate of $2 \mu \mathrm{g} / \mathrm{kg} / \mathrm{min}$ for 2 hours, and then increased to $4 \mu \mathrm{g} / \mathrm{kg} / \mathrm{min}$ for further 22 hours. After the therapy, patients received observation for 5-7 days in hospital. During this period, echocardiography was tested with Simpson's method. Dyspnea and systemic HF symptoms, and NYHA class was assessed. B-type Natriuretic Peptide (BNP) plasma levels were tested. Haemodynamic variables were measured at baseline and at 1, 2, 3, 4, 6, and $24 \mathrm{~h}$ in patients received Swan-Ganz catheterization test at baseline. Measurements included PCWP, CI, Cardiac Output (CO), Pulmonary Average Mean Pressure (PAWP), Stoke Volume (SV) and Systemic Vascular Resistance (SVR). Also lab tests and safety assessment were assessed.

\section{Statistical methods}

Full Analysis Sets (FAS) and Per-Protocol Sets (PPS) were all used in efficacy analyses. Paired-t-test was used to compare the data in the group with normal distribution. Wilcoxon signed rank test was used to compare the data in the group with non-normal distribution. Analysis of covariance adjusted center effects and baseline effects was used to compare the data between groups after test of homogeneity of variance. Cochran mantel-haenszel $\chi 2$ test adjusted center effect was used to compare count data between groups. All statistical analyses were performed using SAS ${ }^{\circ} 91.3$ (SAS Institute, Cary, NC, USA), testing significant resistance levels with bilateral $\alpha=0.05$. All authors made the decision to submit the manuscript for publication and assume responsibility for the accuracy and completeness of the data and analyses.

\section{Results}

Patient baseline characteristics
In patients with or without Swan-Ganz catheterization test, the differences of demographic characteristics including age and gender, and baseline HF characteristics including blood pressure, heart rate (Table 1) and treatment of heart failure medication (including $\beta$ blockers, angiotensin converting enzyme inhibitors or angiotensin receptor antagonists, aldosterone antagonists, diuretics, vasodilators and inotropic agents) between levosimendan group and dobutamine group were not statistically significant. The etiology of heart failure (including ischemic cardiomyopathy, dilated cardiomyopathy and valvular heart disease after surgery) was also no remarkable difference between the two groups.

\section{Echocardiography and haemodynamic assessments between two groups after $24 \mathrm{~h}$}

Echocardiographic assessments: After $24 \mathrm{~h}$ of treatment, LVEF was significantly increased and Left Ventricular End-Systolic Diameter (LVESD) was significantly decreased $(\mathrm{P}<0.01)$ in both groups compared with baselines. SV was significantly increased in levosimendan group $(\mathrm{P}=0.03)$. Left Ventricular End-Diastolic Diameter (LVEDD) was significantly decreased in dobutamine group. Comparing with levosimendan and dobutamine groups, the change rate of LVESD and SV from baselines showed no statistically significant difference between the two groups $(\mathrm{P}>0.05)$ (Figure 1).

Haemodynamic assessments: After $24 \mathrm{~h}$ of treatment, PCWP, CI, CO, PAMP, and SV showed statistically significant differences compared with baselines in both groups (all $\mathrm{P}<0.05$ or $<0.01$ ). The SVR had statistically significant difference compared with baseline only in levosimendan group $(\mathrm{P}<0.01)$. Comparing with levosimendan and dobutamine groups, the change rate of PCWP, CI, SVR and CO from baselines showed statistically significantly difference between the two groups $(\mathrm{P}=0.04, \mathrm{P}=0.01, \mathrm{P}=0.01, \mathrm{P}=0.01)$ (Figure 2).

\section{NT-proBNP assessment between two groups after $72 \mathrm{~h}$}

NT-proBNP levels were significantly decreased when compared with baselines in both groups. The change rate of NT-proBNP from baseline in levosimendan group [(-22.36 \pm 38.98$) \%]$ was significantly greater than which in dobutamine group $[(-8.56 \pm 42.42) \%](\mathrm{P}<0.01)$.

\section{Clinical conditions assessments after treatment}

Improvement of dyspnea: After $24 \mathrm{~h}$ of administration of levosimendan, the proportions of patients with markedly improved dyspnea symptom, improved dyspnea symptom and no improved dyspnea symptom were separately $15.3 \%, 28.8 \%$ and $55.8 \%$. After 24 $\mathrm{h}$ of administration of dobutamine, the proportions of patients with

\begin{tabular}{|c|c|c|c|}
\hline & $\begin{array}{c}\text { Levosimendan } \\
\text { group }(\mathbf{n = 1 1 4})\end{array}$ & $\begin{array}{c}\text { Dobutamine group } \\
(\mathbf{n = 1 1 4 )}\end{array}$ & $\begin{array}{c}\boldsymbol{P} \\
\text { value }\end{array}$ \\
\hline Age (years), mean (SD) & $54.2(12.5)$ & $54.5(12.9)$ & 0.85 \\
\hline Gender male, n (\%) & $88(77.2)$ & $86(75.4)$ & 0.76 \\
\hline BMI (kg/m²), mean (SD) & $23.7(3.5)$ & $23.7(3.4)$ & 0.97 \\
\hline LVEF (\%), mean (SD) & $28.4(7.1)$ & $28.6(7.5)$ & 0.84 \\
\hline SBP (mmHg), mean (SD) & $113.5(16.7)$ & $116.9(20.0)$ & 0.16 \\
\hline DBP (mmH), mean (SD) & $72.6(11.0)$ & $74.1(12.9)$ & 0.35 \\
\hline $\begin{array}{c}\text { HR (beats per minutes), } \\
\text { mean (SD) }\end{array}$ & $78.7(13.7)$ & $78.7(14.4)$ & 0.99 \\
\hline $\begin{array}{c}\text { Breath (per minutes ), } \\
\text { mean (SD) }\end{array}$ & $18.7(3.2)$ & $18.9(3.4)$ & 0.54 \\
\hline
\end{tabular}

BMI: Body Mass Index; SBP: Systolic Blood Pressure; DBP: Diastolic Blood Pressure; HR: Heart Rate

Table 1: Characteristics of the study patients $(n=228)$. 
Citation: Gan T, Zhao X, Huang Y, Zhang Y, Qing E, et al. (2016) A Comparison of Haemodynamic Effects and Safety between Domestic Levosimendan versus Dobutamine for Hospitalized Patients with Acute Decompensated Heart Failure. Cardiovasc Pharm Open Access 5: 186. doi:10.4172/2329-6607.1000186

Page 3 of 5
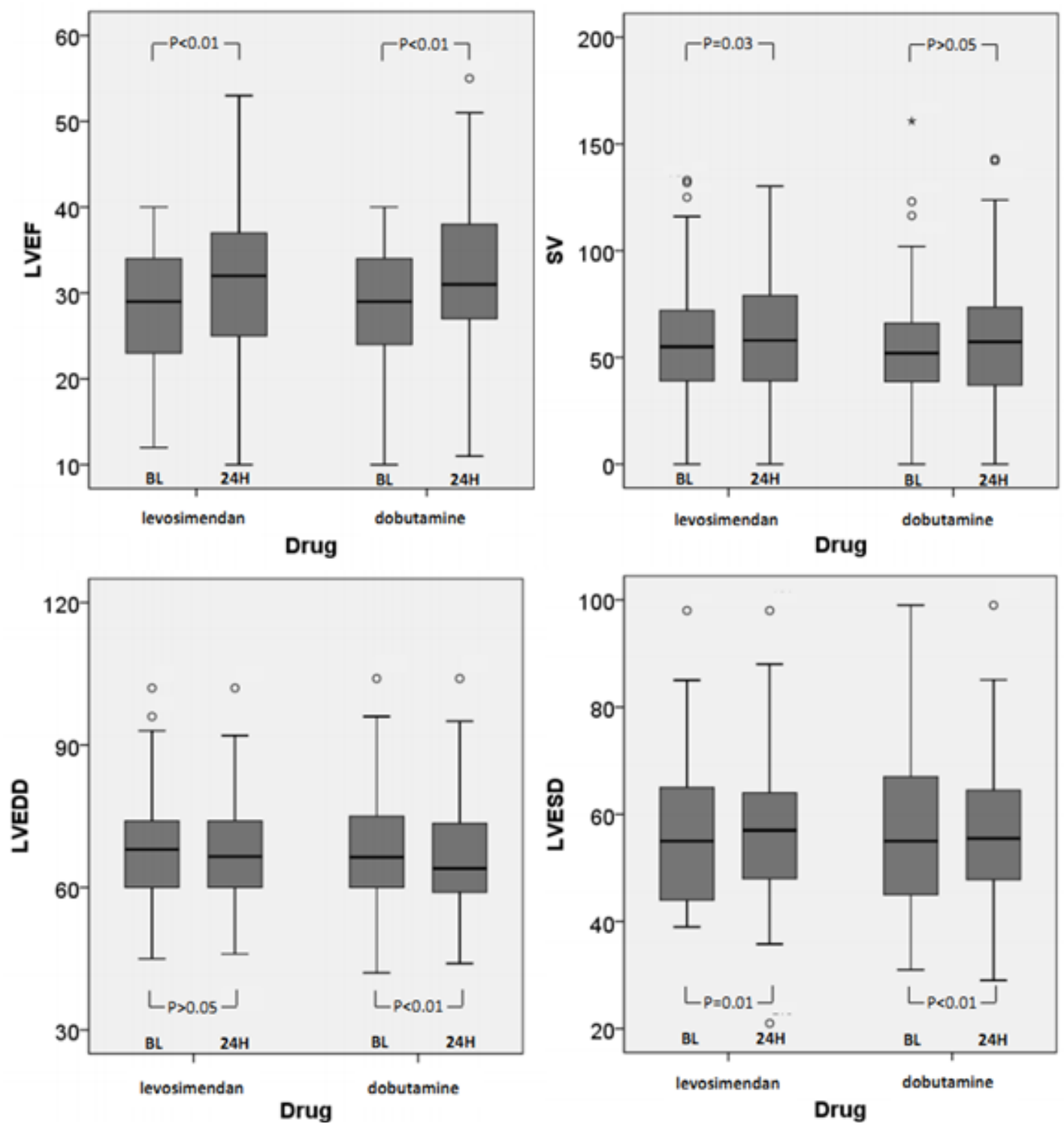

BL: Baselines; $24 \mathrm{H}: 24$ hours

Figure 1: Absolute changes in echocardiographic assessments in levosimendan group and dobutamine group.

markedly improved dyspnea symptom, improved dyspnea symptom and no improved dyspnea symptom were separately $7.21 \%, 15.3 \%$ and $69.3 \%$. There was significant difference between two groups $(\mathrm{P}=0.04)$.

NYHA class assessments: After $24 \mathrm{~h}$ and 5-7 days of administration, there was no significant difference on improvement of NYHA class between levosimendan group and dobutamine group (all $\mathrm{P}>0.05$ ).

Other assessments: After $24 \mathrm{~h}$ of administration, the changes of edema, hepatomegaly, jugular vein distention, lung rales and urine volume of $24 \mathrm{~h}$ from baselines were no significant difference between two groups (all $\mathrm{P}>0.05$ ).

\section{Safety assessments}

Changes in vital signs before and after administration: the systolic and diastolic blood pressure in levosimendan group were significantly lower compared with dobutamine group after 1, 2, 3, 4, 6 and $24 \mathrm{~h}$ of administration (all $\mathrm{P}<0.05$ or $<0.01$ ). The systolic and diastolic blood pressure had no significant difference between two groups on baseline and after 5-7 days of administration. Safety assessments including change of heart rate, arrhythmia, liver and renal function showed no difference before and after administration, and also no difference between two groups (all $\mathrm{P}>0.05$ ).

Adverse events: the incidences of adverse events were $28.07 \%$ in levosimendan group and $38.60 \%$ in dobutamine group. The incidences of side effects were $7.02 \%$ in levosimendan group and $8.77 \%$ in dobutamine group. The incidences of severe adverse events were $1.75 \%$ in levosimendan group and $3.51 \%$ in dobutamine group. The incidences of severe side effects were $0.88 \%$ in levosimendan group and $1.75 \%$ in dobutamine group. There was no significant difference in all these between two groups. 
Citation: Gan T, Zhao X, Huang Y, Zhang Y, Qing E, et al. (2016) A Comparison of Haemodynamic Effects and Safety between Domestic Levosimendan versus Dobutamine for Hospitalized Patients with Acute Decompensated Heart Failure. Cardiovasc Pharm Open Access 5 : 186. doi:10.4172/2329-6607.1000186

Page 4 of 5
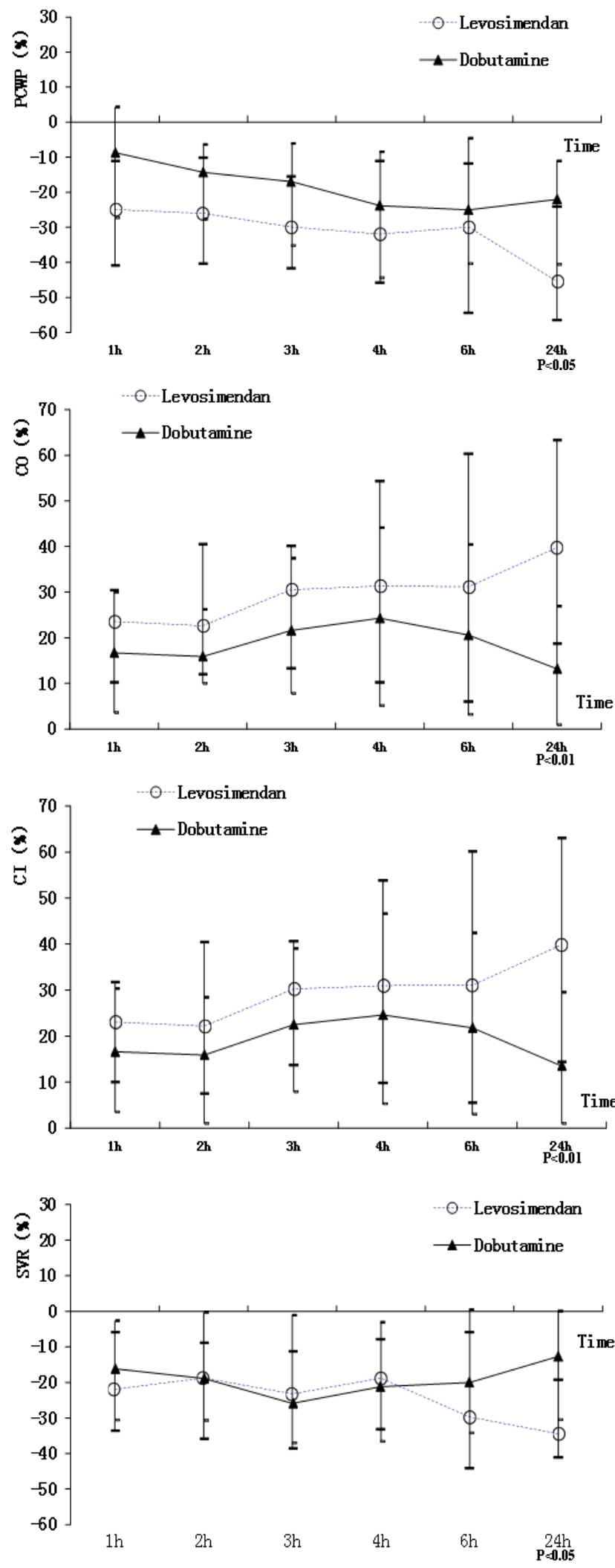

Figure 2: Change rates of haemodynamic assessments in levosimendan group and dobutamine group.

\section{Discussion}

Levosimendan is a novel calcium sensitizer, which has inotropic effect by increasing sensitivity of $\mathrm{Ca}^{2+}$ in the contraction site. Levosimendan could improve myocardial contractility without increasing intracellular cyclic Adenosine Monophosphate (cAMP) or $\mathrm{Ca}^{2+}$ concentration. But levosimendan does not affect heart rate and increase myocardial oxygen consumption. In addition, the inotropic effect of levosimendan is not affected by $\beta$ blockers, so that levosimendan could be used accompanied by $\beta$ blockers [2]. The LIDO study showed that levosimendan may improve the hemodynamic of acute heart failure and 180-day survival rate more effectively [3]. García-González et al. founded that levosimendan can improve haemodynamic parameters in critically ill patients with reduce LVEF [4].

This was a multi-center, randomized, blinded, phase II clinical trial comparing efficacy and safety of domestic levosimendan with dobutamine. Levosimendan showed a significantly greater improvement in LVEF and a significantly greater decrease in LVESD compared with dobutamine. The data from patient with Swan-Ganz catheterization showed levosimendan had significantly greater decreases in PCWP and SVR, and significantly greater increases in CO and CI after $24 \mathrm{~h}$ treatment of levosimendan compared with dobutamine. In general, levosimendan could do better in improving hemodynamics in ADHF patients, improving cardiac output while reducing pulmonary congestion, and reducing the circulation resistance. Previous study had shown that levosimendan also could improve hemodynamics in patients with chronic heart failure, increase cardiac contractility and dilate blood vessels [5].

After $72 \mathrm{~h}$ treatment of levosimendan, NT-proBNP level was significantly reduced. This reduction occurred after 3 days of treatment, which may related to the peak time of bioactive metabolite of levosimendan on the third day. The significant reduction of NTproBNP level may partially reflect the treatment effect on acute heart failure. The value and rate of change of NT-proBNP level after 3 day treatment of levosimendan from baseline were significantly greater than which in dobutamine group, indicating that levosimendan had better effect on relieving acute heart failure than dobutamine. The SURVIVE study [6] enrolled 1327 cases of acute heart failure patients, and the results showed that levosimendan had significantly greater decrease in BNP level after administration of 24 hours than dobutamine. Research by Parissis [7] showed that levosimendan significantly reduced NTproBNP level and tumor necrosis factor- $\alpha$ level, but no such changing in dobutamine group before and after treatment.

In the aspect of clinical symptoms, levosimendan group had significantly greater improvement of dyspnea than dobutamine group, which also because of their rapidly reduction of PCWP and increasing of CO. There were also some improvements in NYHA class and rales, however there was no significant difference between levosimendan and dobutamine.

In the safety evaluation, after administration of levosimendan, systolic and diastolic blood pressure of patients were significantly reduced on 1, 2, 3, 4 and 24 hours compared with dobutamine. But the lowest average blood pressure was $106.9 \mathrm{mmHg}$ over $66.21 \mathrm{mmHg}$, in the safe level of absence of any clinical complaints. The haemodynamic monitoring also showed SVR reduced after treatment of levosimendan, which meant levosimendan could dilate blood vessels while decreasing PCWP and increasing CO, thereby reducing afterload in acute heart failure patients. The pharmacological effect is that levosimendan could 
Citation: Gan T, Zhao X, Huang Y, Zhang Y, Qing E, et al. (2016) A Comparison of Haemodynamic Effects and Safety between Domestic Levosimendan versus Dobutamine for Hospitalized Patients with Acute Decompensated Heart Failure. Cardiovasc Pharm Open Access 5: 186. doi:10.4172/2329-6607.1000186

Page 5 of 5

activate the ATP-dependent potassium channel. Safety evaluation including heart rate, liver and renal function have no significant difference before and after treatment. There was no statistically remarkable difference in the incidence of overall adverse events between the two groups. However, dobutaine group has significantly higher incidence of arrhythmias than levosimendan group, which may be linked with increased myocardial oxygen consumption caused by activation of dopamine receptor and increasing of calcium influx. Levosimendan does not increase the incidence of malignant arrhythmia.

In summary, the results of this study revealed that the domestic levosimendan had better effects on reducing PCWP, increasing CO and reducing SVR than dobutamine. Also, levosimendan could lower NTproBNP level and improve dyspnea with good tolerability and safety.

\section{Funding}

This study was supported by grants from the Key Projects in the National Science \& Technology Pillar Program of the 12th Five-year Plan Period (No.2011BA111B02), project for heart failure, Beijing, China.

\section{References}

1. Rognoni A, Lupi A, Lazzero M, Bongo AS, Rognoni G (2011) Levosimendan from basic science to clinical trials. Recent Pat Cardiovasc Drug Discov 6: 9-15

2. Innes CA, Wagstaff AJ (2003) Levosimendan: a review of its use in the management of acute decompensated heart failure. Drugs 63: 2651-2671.

3. Follath F, Cleland JG, Just H, Papp JG, Scholz H, et al. (2002) Efficacy and safety of intravenous levosimendan compared with dobutamine in severe lowoutput heart failure (the LIDO study): a randomized double-blind trial. Lancet 360: 196-202.

4. García-González MJ, Jorge-Pérez $P$, Jiménez-Sosa $A$, Acea AB, Lacalzada Almeida JB, et al. (2015) Levosimendan improves hemodynamic status in critically ill patients with severe aortic stenosis and left ventricular dysfunction: an interventional study. Cardiovasc Ther 33: 193-199.

5. Nieminen MS, Akkila J, Hasenfuss G, Kleber FX, Lehtonen LA, et al (2000) Hemodynamic and neurohumoral effects of continuous infusion of levosimendan in patients with congestive heart failure. J Am Coll Cardiol 36: 1903-1912.

6. Mebazaa A, Nieminen MS, Packer M, Cohen-Solal A, Kleber FX, et al. (2007) Levosimendan vs dobutamine for patients with acute decompensated heart failure: the SURVIVE randomized trial. JAMA 297: 1883-1891.

7. Parissis JT, Panou F, Farmakis D, Adamopoulos S, Filippatos G, et al. (2005) Effects of levosimendan on markers of left ventricular diastolic function and neurohormonal activation in patients with advanced heart failure. Am J Cardiol 96: 423-426. 